\title{
Multilevel regulation of Wnt signaling by Zic2 in colon cancer due to mutation of $\beta$-catenin
}

\author{
Zhengshui Xu', Jianbao Zheng ${ }^{1}$, Zilu Chen ${ }^{1}$, Jing Guo ${ }^{1}$, Xiaopeng Li', Xingjie Wang $\mathbb{D}^{1}$, Chao Qu', Liyue Yuan², \\ Chen Cheng ${ }^{1}$, Xuejun Sun ${ }^{1}$ and Junhui $\mathrm{Yu}^{1}$
}

\begin{abstract}
Zinc-finger of the cerebellum 2 (Zic2) is widely implicated in cancers, but the role of Zic2 in tumorigenesis is bilateral. A recent study indicated that Zic2 could render colon cancer cells more resistant to low glucose-induced apoptosis. However, the functional roles of Zic2 in colon cancer and the underlying molecular mechanism remain elusive. Herein, we demonstrated that Zic2 was highly expressed in colon cancer tissues and correlated with poor survival. Knockdown of Zic2 inhibited colon cancer cell growth, arrested the cell cycle transition from G0/G1 to S phase, and suppressed tumor sphere formation in vitro; in addition, silencing Zic2 retarded xenograft tumor formation in vivo. Consistently, ectopic expression of Zic2 had the opposite effects. Mechanistically, Zic2 executed its oncogenic role in colon cancer by enhancing Wnt/ $\beta$-catenin signaling. Zic2 directly binds to the promoter of Axin2 and transcriptionally represses Axin2 expression and subsequently promotes the accumulation and nuclear translocation of $\beta$-catenin. Meanwhile, Zic2 could activate Wnt signaling by interacting with $\beta$-catenin. Intriguingly, in HCT116 cells with intrinsic Ser45 mutation of $\beta$-catenin, which blocks the degradation-related phosphorylation of $\beta$-catenin by CK1, modified Zic2 expression did not affect the protein level of $\beta$-catenin. Altogether, our findings uncover a novel multilevel mechanism for the oncogenic activity of Zic2 in colon cancer and suggest Zic2 as a potential therapeutic target for colon cancer patients.
\end{abstract}

\section{Background}

Colorectal cancer (CRC) is the second most prevalent cancer and the fourth leading cause of cancer-related lethality globally ${ }^{1}$. Approximately $90 \%$ of CRCs have abnormal Wnt signaling activation, which plays important role in cancer stem cell (CSC) biology, tumorigenesis, and metastasis in $\mathrm{CRC}^{2-5}$. Nuclear translocation of $\beta$-catenin is considered the hallmark of the canonical Wnt pathway $^{2,4,6}$. The disruption of the $\beta$-catenin destruction complex, consisting of $\mathrm{CK} 1^{7,8}, \mathrm{GSK}-3 \beta^{9,10}, \mathrm{Axin}^{7,11}$, and $\mathrm{APC}^{10,12}$, results in the activation of Wnt signaling. Among the $\beta$-catenin destruction complex, AXIN2,

\footnotetext{
Correspondence: Xuejun Sun (sunxy@mail.xjtu.edu.cn) or

Junhui Yu (yujunhui@mail.xjtu.edu.cn)

${ }^{1}$ Department of General Surgery, First Affiliated Hospital of Xi'an Jiaotong

University, Xi'an 710061 Shaanxi Province, PR China

${ }^{2}$ Department of Endocrinology, First Affiliated Hospital of Xi'an Jiaotong

University, Xi'an 710061 Shaanxi Province, PR China

These authors contributed equally: Zhengshui Xu, Jianbao Zheng

Edited by E. Candi
}

playing crucial role in $\mathrm{Wnt} / \beta$-catenin signaling pathway, participates in regulating cell proliferation, apoptosis, migration, and other important functions in the development of various cancers ${ }^{13}$. It is generally accepted that CK1, by binding to Axin2, initiates $\beta$-catenin degradation by phosphorylation at Ser45; subsequently, GSK-3 $\beta$ catalyzes the phosphorylation of $\beta$-catenin at more $\mathrm{N}$ terminal residues (Ser33, Ser37, and Thr41) $)^{5-7,14}$, while APC can intensify the affinity of phosphorylases for $\beta$-catenin to accelerate $\beta$-catenin degradation in the cytoplasm via the ubiquitin-proteasome ${ }^{2,6}$. Dephosphorylated $\beta$-catenin accumulated in the cytoplasm can translocate into the nucleus and form complexes with member(s) of the TCF/LEF family to initiate transcription of downstream target genes, such as cyclin D1, CD44, cMyc, and Lgr $5^{15-18}$. Mutations in $\mathrm{APC}^{5,19}, \beta$-catenin ${ }^{2,14}$, and Axin $1^{17}$ in CRC block the destruction complex's affinity for $\beta$-catenin, which triggers aberrant accumulation of $\beta$-catenin. Among colon cancer cell lines, HCT116

\section{(c) The Author(s) 2021}

(c) (i) Open Access This article is licensed under a Creative Commons Attribution 4.0 International License, which permits use, sharing, adaptation, distribution and reproduction cc) in any medium or format, as long as you give appropriate credit to the original author(s) and the source, provide a link to the Creative Commons license, and indicate if changes were made. The images or other third party material in this article are included in the article's Creative Commons license, unless indicated otherwise in a credit line to the material. If material is not included in the article's Creative Commons license and your intended use is not permitted by statutory regulation or exceeds the permitted use, you will need to obtain permission directly from the copyright holder. To view a copy of this license, visit http://creativecommons.org/licenses/by/4.0/. 
has a special three-base deletion in the $\beta$-catenin encoding gene, CTNNB1, causing an in-frame deletion at Ser $45^{20}$.

Zinc-finger protein of the cerebellum 2 (Zic2), homologous to the Drosophila protein odd-paired, belongs to the Zic family of proteins, which contain highly conserved $\mathrm{C} 2 \mathrm{H} 2$ zinc-finger motifs ${ }^{21-23}$. As a transcription factor, Zic2 plays important role in cerebral formation in both animals and humans ${ }^{24,25}$. Mutations in Zic2 cause spina bifida and axial and limb bone malformation ${ }^{23}$. Likewise, Zic2 functions in tumorigenesis ${ }^{26}$, cancer stem cell renewal $^{21}$, proliferation ${ }^{27-31}$, invasion, and metas$\operatorname{tasis}^{28,30,32}$. However, the functions and mechanisms of Zic2 in various cancers are extraordinarily complicated and remain unclear ${ }^{29}$. In CRC, only one Zic2-related study has been published, in which Zhao et al. reported that Zic2 can render colon cancer cells more resistant to low-glucose-induced apoptosis ${ }^{31}$. However, how Zic2 regulates CRC tumorigenesis and progression remains elusive.

\section{Materials and methods Clinical samples}

A tissue microarray of 180 pairs of primary CRC tissues was purchased from Shaanxi Kexin Biotechnology Co., Ltd (cohort I). Four hundred and three colon cancer tissue samples and paired normal colon tissue samples were randomly selected from CRC patients who had not received radiotherapy or chemotherapy before excision between January 2011 and December 2016 (cohort II). All the patients underwent surgery at the First Affiliated Hospital of Xi'an Jiaotong University. Informed consent forms were signed by all patients. Our study protocol was approved by the Ethics Committee of the First Affiliated Hospital of Xi'an Jiaotong University.

\section{Lentiviral vectors and transfection}

Lentiviral vectors with Zic2 shRNA or Zic2 overexpression were purchased from GeneChem Co., Ltd. (Shanghai, China). The target short hairpin RNA (shRNA) sequences were presented in Supplementary Table 3. Lentiviral infection referred to the manufacturer's protocol.

\section{Immunohistochemistry (IHC)}

For IHC, the staining procedure was performed using the standard avidin-biotin complex method. The extent of positively stained cells was scored as previously described $^{33}$. The Zic2-stained (Avia Systems Biology, San Diego, CA, USA) sections were divided into two groups (High and Low) based on scores of the staining between colon cancer tissues and adjacent colon normal tissues. Two pathologists evaluated all the specimens in a blinded manner.

\section{RNA isolation and real-time PCR}

Total RNA was isolated from cells using TRIzol reagent (Invitrogen, Carlsbad, CA, USA). Complementary DNA (ctDNA) was synthesized by the PrimeScript RT Reagent Kit (TaKaRa, Osaka, Japan). Real-time PCR was conducted on an IQ5 instrument (Bio-Rad, CA, USA) using SYBR Green fluorescence signal detection assays (TaKaRa, Osaka, Japan) with primers list in Supplementary Table 4 . The specific mRNA expression level was quantified by using the $2-\Delta \Delta \mathrm{CT}$ method $^{33}$.

\section{Luciferase reporter assay}

For promoter analyses, a fragment of the Axin2 5'flanking sequence (from -912 to $+207 \mathrm{bp}$ ) and other truncated fragments were cloned into the pGL3.0 Basic Vector (Promega, Madison, WI, USA) to generate a Axin2 full promoter reporter construct and the truncated ones (Supplementary Table 1). The plasmids containing firefly luciferase reporters of PTEN promoter and the truncated ones and the pTK-RL plasmids were co-transfected into cells. The detailed protocol was carried out as described previously $^{33}$.

\section{Quantitative chromatin immunoprecipitation (qChIP)}

The qChIP assay was conducted using the EZ-ChIP Kit (Millipore, Bedford, MA, USA) according to the method of the manufacturer's instructions ${ }^{33} .5 \mu \mathrm{g}$ anti-Zic2 antibody and $1 \mu \mathrm{g}$ IgG negative control antibody were used to precipitate the chromatin-protein mixture. Finally, the target fragment or endogenous non-coding region fragment were amplified with specific primers (Supplementary Table 1) by using real-time PCR.

\section{Cell cultures}

DLD-1, HCT116, and SW480 cells (Shanghai Institute of Cell Biology, Chinese Academy of Sciences) were authenticated by STR analysis (GeneChem Co., Ltd., Shanghai, China). Cell lines were maintained in DMEM and McCoy's 5A medium (HyClone, Logan, UT, USA) supplemented with 10\% FBS (Gibco BRL, Carlsbad, CA, USA) and 100 units $/ \mathrm{ml}$ of penicillin and streptomycin at $37^{\circ} \mathrm{C}$ in a humidified $5 \% \mathrm{CO}_{2}$ atmosphere.

\section{Immunohistochemistry (IHC)}

For IHC, the staining procedure was performed using the standard avidin-biotin complex method. The extent of positively stained cells was scored as previously described $^{33}$. The Zic2-stained (Avia Systems Biology, San Diego, CA, USA) sections were divided into two groups (High and Low) based on scores of the staining between colon cancer tissues and adjacent colon normal tissues. Two pathologists evaluated all the specimens in a blinded manner. 


\section{Tumor spheres}

Cells were counted and plated in SFM [DMEM/F12 medium (Thermo Scientific, Mulgrave, Australia), 1\% B27 supplement without vitamin A (Thermo Scientific), $20 \mathrm{ng} / \mathrm{ml}$ EGF (Thermo Scientific), and $20 \mathrm{ng} / \mathrm{ml} \mathrm{FGF}$ (R\&D Systems, Minneapolis, MN)] in ultralow attachment six-well plates (Corning, Tewksbury, MA) at a total of $1.5 \times 10^{3}$ cells per well, and cultured for up to 2 weeks to allow for spheroid growth. The medium was refreshed every 3 days.

\section{Cell counting kit-8 (CCK8) assay}

Cells were incubated at $5 \% \mathrm{CO}_{2}$ and $37^{\circ} \mathrm{C}$ on 96 -well plates $(100 \mu \mathrm{L} /$ well). Ten microliters of CCK-8 reagent (Dojindo, Kyushu, Japan) was added to each well after 24, 48,72 , and $96 \mathrm{~h}$, respectively. OD450 values were determined by a microplate reader.

\section{Colony formation assay}

Cells were plated into $60-\mathrm{mm}$ plates and routinely cultured for 14 days. The cells were subsequently fixed with methyl alcohol for $15 \mathrm{~min}$ and stained with $0.1 \%$ crystal violet.

After staining, the visible colonies were counted.

\section{Cell cycle assay}

Cells were harvested and fixed in $75 \%$ cold ethanol and stored at $4{ }^{\circ} \mathrm{C}$ overnight. The next day cells were centrifuged at $1500 \mathrm{rpm}$ for $5 \mathrm{~min}$. After treatment with RNase A at $37^{\circ} \mathrm{C}$ for $30 \mathrm{~min}$, cell pellets were further washed with PBS and stained with PI $(10 \mu \mathrm{g} / 100 \mu \mathrm{l})$ for $10 \mathrm{~min}$ in the dark. The cell cycle was assessed with flow cytometry (Beckman Coulter).

\section{Nude mouse xenograft assay}

The use of all animals in this study was approved by the Institutional Animal Care and Use Committee of the First Affiliated Hospital of Xi'an Jiaotong University. Tumor cells $\left(5 \times 10^{6}\right)$ in logarithmic phase were sub-cutaneously injected into the right flanks of 5-week-old female BALB/ c-nude mice (Shanghai SLAC Laboratory Animal Co. Ltd., Shanghai, China). After 1 week post-injection, the length $(a)$ and width $(b)$ of the tumor were monitored using callipers every 3 days. The tumor volume $(V)$ was calculated as follows: $V=a b^{2} / 2$. Three weeks later, the mice were sacrificed and the xenograft tumors were measured.

\section{Protein extraction and western blotting}

Cells were lysed using RIPA buffer (Heart, Xian, China). Cell lysates containing $30 \mu \mathrm{g}$ of total protein were then subjected to SDS-PAGE (Beyotime, Shanghai, China) and then transferred to PVDF membranes (Millipore, Billerica, MA, USA). The membranes were incubated with primary antibodies overnight at $4{ }^{\circ} \mathrm{C}$ (anti-Zic2, Axin2, APC, active- $\beta$-Catenin(ser45), $\beta$-Catenin, Flag, and cMyc, 1:1000 dilution; Cyclin D1 and GAPDH, 1:5000). The membrane was then washed six times with TBST buffer for $5 \mathrm{~min}$ each and incubated with a horseradish peroxidase-conjugated secondary antibody at room temperature for $1 \mathrm{~h}$. Chemiluminescent HRP substrate (Millipore, Billerica, MA, USA) was added to visualize the protein bands. The antibodies against GAPDH were purchased from Santa Cruz (Dallas, TX, USA), the antibodies against Zic2, Cyclin D1, CD44, Axin2, and APC were purchased from Abcam (Cambridge, MA). The antibodies against active- $\beta$-Catenin(ser45), $\beta$-Catenin, and GSK-3 $\beta$ were purchased from Cell Signaling Technology (Danvers, MA, USA), and the antibodies against Flag was purchased from Proteintech (Rosemont, USA). Nuclear extract was prepared with the protocol in the Nuclear Extraction Kit (Abcam, Cambridge, MA, USA).

\section{RNA-seq analysis}

The sequencing data was filtered with SOAPnuke (v1.5.2) ${ }^{34}$ by (1) Removing reads containing sequencing adapter; (2) Removing reads whose low-quality base ratio (base quality less than or equal to 5) is more than 20\%; (3) Removing reads whose unknown base (' $N$ ' base) ratio is more than 5\%, afterward clean reads were obtained and stored in FASTQ format. The clean reads were mapped to the reference genome using HISAT2 (v2.0.4) ${ }^{35}$. Bowtie2 $(\mathrm{v} 2.2 .5)^{36}$ was applied to align the clean reads to the reference coding gene set, then expression level of gene was calculated by RSEM (v1.2.12) ${ }^{37}$. The heatmap was drawn by pheatmap $(\mathrm{v} 1.0 .8)^{38}$ according to the gene expression in different samples. Essentially, differential expression analysis was performed using the DESeq2 $(\mathrm{v} 1.4 .5)^{39}$ with $Q$ value $\leq 0.05$. To take insight to the change of phenotype, GO (http://www.geneontology.org/) analysis of annotated different expressed gene was performed by Phyper (https://en.wikipedia.org/wiki/ Hypergeometric_distribution) based on Hypergeometric test. The significant levels of terms and pathways were corrected by $Q$ value with a rigorous threshold ( $Q$ value $\leq$ 0.05 ) by Bonferroni ${ }^{40}$.

\section{Co-immunoprecipitation (Co-IP) assays}

Cells were lysed in Triton X-100 lysis buffer $[\mathrm{NaCl}$ $(150 \mathrm{mM}), \mathrm{NP}-40(0.5 \%)$, Tris- $\mathrm{HCl}(\mathrm{pH}=8.0)$, glycerol (20 mM, 20\%)] containing phosphatase inhibitors and protease inhibitor (Roche, NJ, USA), then the proteins extract were centrifugated at $12,000 \times g$ for $20 \mathrm{~min}$. Thermo Scientific Pierce Co-IP kit (Thermo Fisher Scientific) was used for Co-IP experiments. At last, obtained proteins were resuspended in $5 \times$ SDS sample loading buffer, heated to $100{ }^{\circ} \mathrm{C}$ for $8 \mathrm{~min}$, and tested by $10 \%$ SDS-polyacrylamide gel electrophoresis (PAGE). 


\section{Immunofluorescence (IF)}

The cells were washed three times with PBS for 10 min each, fixed with $4 \%$ paraformaldehyde for $20 \mathrm{~min}$, and permeabilized with $0.2 \%$ Triton X-100 for $10 \mathrm{~min}$. After blocking with $5 \%$ bovine serum albumin (BSA) for $30 \mathrm{~min}$ at room temperature, the cells were incubated at $4{ }^{\circ} \mathrm{C}$ overnight with primary antibodies against Zic2 (1:100 dilution). The dishes were washed three times with PBS for $10 \mathrm{~min}$ each and then incubated with Alexa Fluor 594conjugated secondary antibodies (1:400 dilution, Invitrogen, Carlsbad, CA, USA) for $1 \mathrm{~h}$ at room temperature. The nuclei were stained with DAPI $(10 \mathrm{mg} / \mathrm{ml})$ for $10 \mathrm{~min}$. The samples were examined via microscopy (Leica Microsystems, Heidelberg, Germany) to analyze the expression and nuclear location of Zic2.

\section{Statistical analysis}

All data are presented as the mean \pm standard deviation (SD). The chi-square test or ANOVA was used to analyze the differences among groups. Correlations were analyzed using Pearson linear-regression analysis. OS and RFS rates were plotted using the Kaplan-Meier method and compared with log-rank test. Multivariate statistical analysis was performed using a Cox regression model. All statistical analyses were performed using SPSS 22.0 software (SPSS Inc., Chicago, IL, USA). $P<0.05$ was defined as statistically significant.

\section{Results}

Zic2 is upregulated in colon cancer samples, and high Zic2 expression correlates with unfavorable survival of colon cancer patients

To explore the role of Zic2 in colon cancer, we first analyzed the expression of Zic2 between colon cancer and normal colon tissues by bioinformatic analysis. The results showed that Zic2 transcript levels were upregulated in colon cancer compared with normal colon tissues in the GSE44706 mRNA microarray dataset (Fig. 1a) and TCGA RNA-sequencing data (Fig. 1b). Zic2 might be a potential prognostic marker in colon cancer because higher Zic2 transcript levels were strongly correlated with poorer overall survival in TCGA data (Fig. 1c).

To confirm the role of Zic2 proteins in colon cancer, we performed immunohistochemical (IHC) staining of 180 colon cancer samples via a tissue microarray (Fig. 1d-f, cohort I) and found a markedly increased protein level of Zic2 in colon cancer tissues compared with adjacent colon normal tissues $(P<0.0001$, Fig. $1 d$, e). Zic2 protein was mainly localized in the nucleus of cells in colon cancer tissues (Fig. 1d) and colon cancer cell lines (Supplementary Fig. 1a). High expression of Zic2 protein was correlated with poorer overall survival (OS) than low expression of Zic2 in colon cancer patients (Fig. 1f). To further validate the role of Zic2 in colon cancer, we performed IHC staining using another independent cohort of 403 colon cancer patient samples (Fig. 1g-i, cohort II). The results were consistent with those observed in cohort I. Moreover, higher Zic2 protein expression was significantly associated with a poorer tumor phenotype, including larger tumor size, a higher rate of stage T3/T4 tumors, and higher AJCC stage (Supplementary Table 1). By multivariate analysis, we found that Zic2 was an independent prognostic factor for poor OS in colon cancer patients (Supplementary Table 2). Altogether, these data indicate that Zic2 is upregulated in colon cancer samples and that high Zic2 expression correlates with unfavorable survival of colon cancer patients.

\section{Zic2 mediates colon cancer cell proliferation by inducing a transition from $\mathrm{G} 0 / \mathrm{G} 1$ to $S$ phase in vitro}

The expression of Zic2 was measured in six established colon cancer cell lines and one human colonic epithelial cell line $(\mathrm{HCoEpiC})$ by western blotting and qRT-PCR analysis (Supplementary Fig. 1b, c). We found that Zic2 transcript levels were higher in all six colon cancer cell lines than in $\mathrm{HCoEpiC}$. To further gain insight into the impact of Zic2 in colon cancer, a series of in vitro and in vivo experiments were performed in colon cancer cells with silenced or overexpressed Zic2. Silencing of Zic2 in DLD-1 and HCT116 cells and ectopic expression of Zic2 in DLD, HCT116, and SW480 cells were validated by western blotting and qRT-PCR analysis (Supplementary Fig. 1d-j).

To investigate the function of Zic2 in vitro, CCK-8 and colony formation assays were performed. As shown in Fig. 2a-d, knockdown of Zic2 repressed the viability and colony formation of DLD-1 and HCT116 cells. Conversely, DLD, HCT116, and SW480 cells with Zic2 overexpression exhibited increased viability and colony formation (Fig. $2 \mathrm{e}-\mathrm{j}$ ). We then attempted to reveal the potential mechanism by which Zic2 mediates colon cancer cell proliferation by analyzing the cell cycle and apoptosis. The results showed that depletion of Zic2 arrested DLD-1 and HCT116 cells in the G0/G1 phase (Fig. 2k, l, Supplementary Fig. 2a-d). Conversely, ectopic expression of Zic2 induced a transition from G0/G1 to $\mathrm{S}$ phase in DLD-1, HCT116, and SW480 cells (Fig. 2m-o, Supplementary Fig. 2e-k). Intriguingly, modulation of Zic2 expression did not affect the apoptosis rate in colon cancer cells (Supplementary Fig. 3). Collectively, these data indicate that Zic2 promotes colon cancer proliferation by inducing a transition from G0/G1 to $\mathrm{S}$ phase rather than by impacting apoptosis.

\section{Zic2 correlates with colon CSC properties}

This is the first analysis of the role of Zic2 in colon CSCs. To test the role of Zic2 in colon CSCs, colon cancer cells with modified Zic2 expression were cultured in 
a

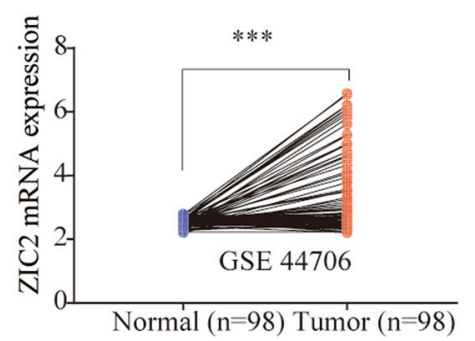

d

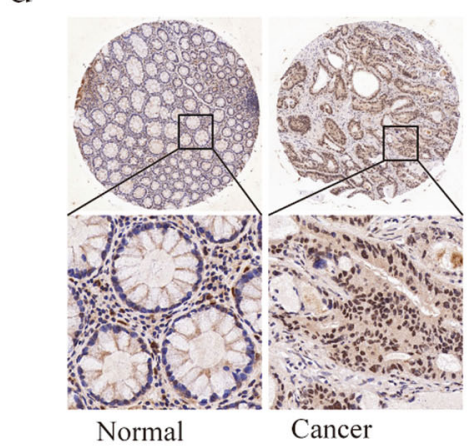

g

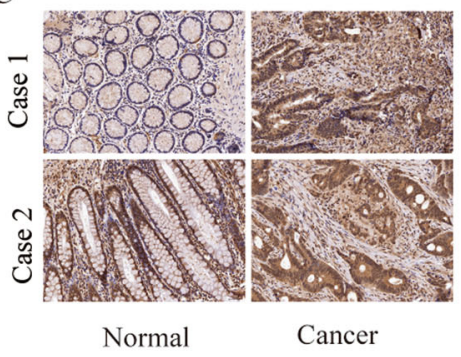

$\mathrm{b}$

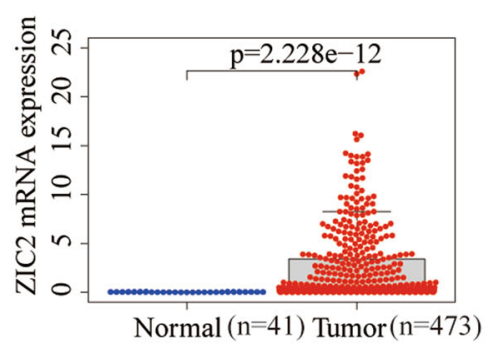

e

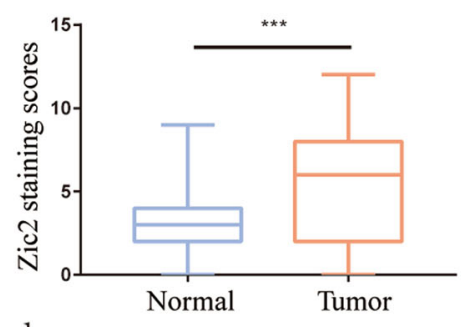

h

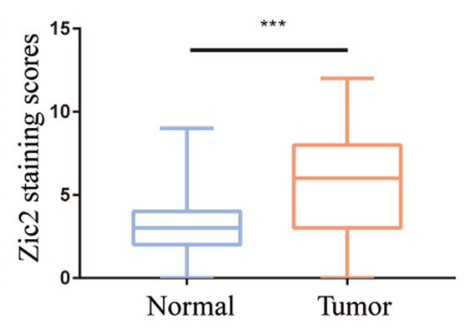

$\mathrm{C}$

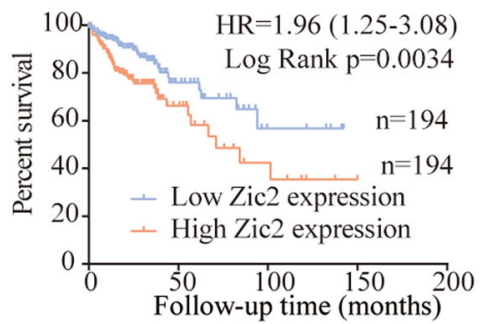

f
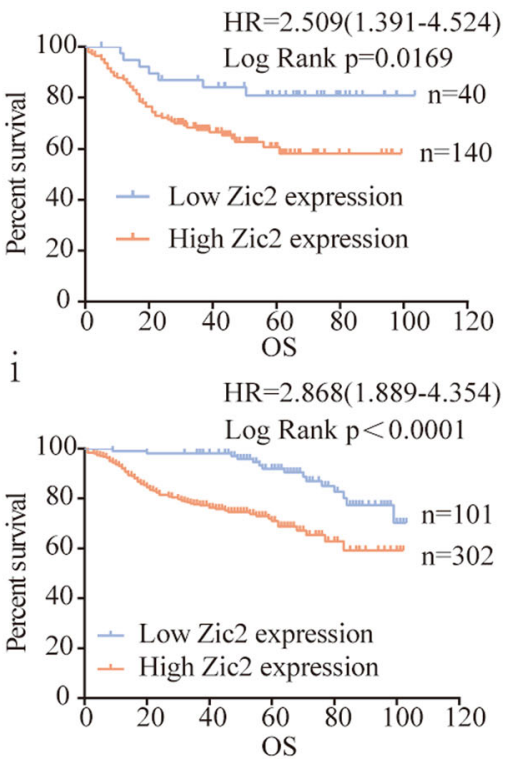

Fig. 1 The expression of Zic2 in colon cancer tissue samples and normal tissue samples. a Zic2 mRNA expression of 98 colon cancer tissue samples and paired NC tissue samples in GSE 44706 mRNA microarray. b Zic2 transcription expression of 473 colon cancer tissue samples and 41 NC tissue samples in TCGA database. c Data in TCGA database showed the overall survival of the two groups of patients with high ( $n=194$, red line) or low $(n=194$, blue line) Zic2 expression in colon cancer tissues. $\mathbf{d}$, $\mathbf{g}$ Representative images of the IHC staining of Zic2 in two independent cohorts (Cohort I (d), $N=180$; Cohort II (g), $N=403$ ). e, $\mathbf{h}$ The staining scores of the Zic2 expression in two independent cohorts. $\mathbf{f}$, $\mathbf{i}$ Kaplan-Meier representation of the overall survival between the two groups of patients with high (red line) and low (blue line) Zic2 expression in two independent cohorts. All data are the mean \pm SD of three independent experiments. ${ }^{* *} P<0.0001$.

anchorage-independent conditions to form tumor spheres. We observed that the size and number of spheres in Zic2-silenced colon cancer cells were remarkably decreased compared with those in control cells (Fig. 3). In addition, enhancing Zic2 expression in DLD, HCT116, and SW480 cells increased the size and number of spheres (Fig. 3). These results established that Zic2 might promote tumor formation and proliferation through the maintenance of colon CSC properties.

Zic2 enhances Wnt signaling activity in colon cancer cells

To further reveal the underlying mechanisms by which Zic2 promotes colon tumor growth, RNA sequencing of three DLD-1-Zic2 cell lines and three DLD-1 control cell lines was performed (Supplementary Fig. 4a). Based on a $Q$ value $<0.05$, there were 930 differentially expressed genes (DEGS), including 422 downregulated and 508 upregulated genes in ectopically expressed Zic2 clones (Supplementary Fig. 4b, c). The above results suggest that Zic2 may play both transcriptional coactivator and transcriptional repressor roles in colon cancer. Gene Ontology (GO) biological process (BP) analysis confirmed that Zic2 promotes colon tumor growth (Supplementary Fig. 4d).

A recent study indicated that Zic2 can render colon cancer cells more resistant to low-glucose-induced apoptosis by activating Wnt signaling ${ }^{31}$. Our results 


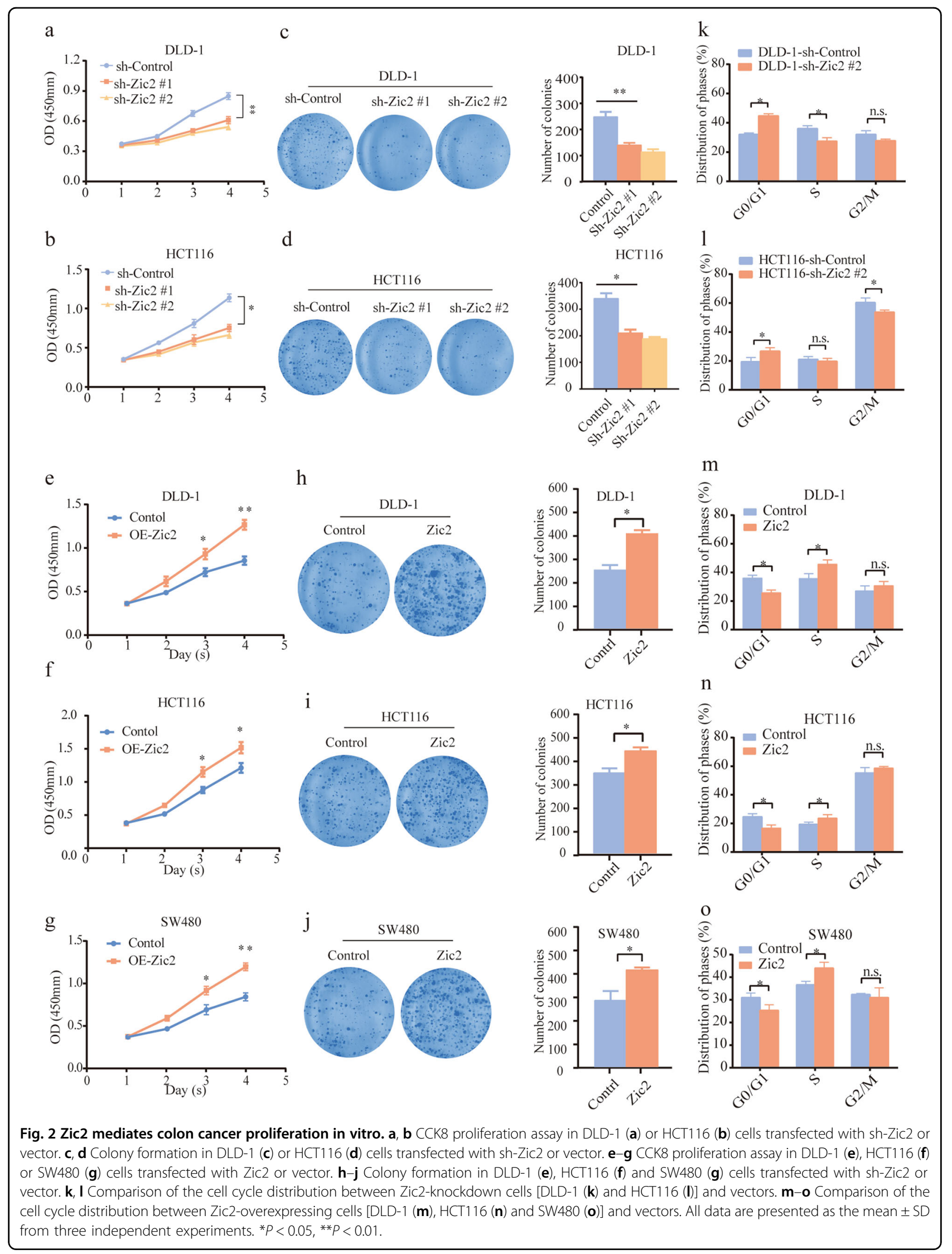




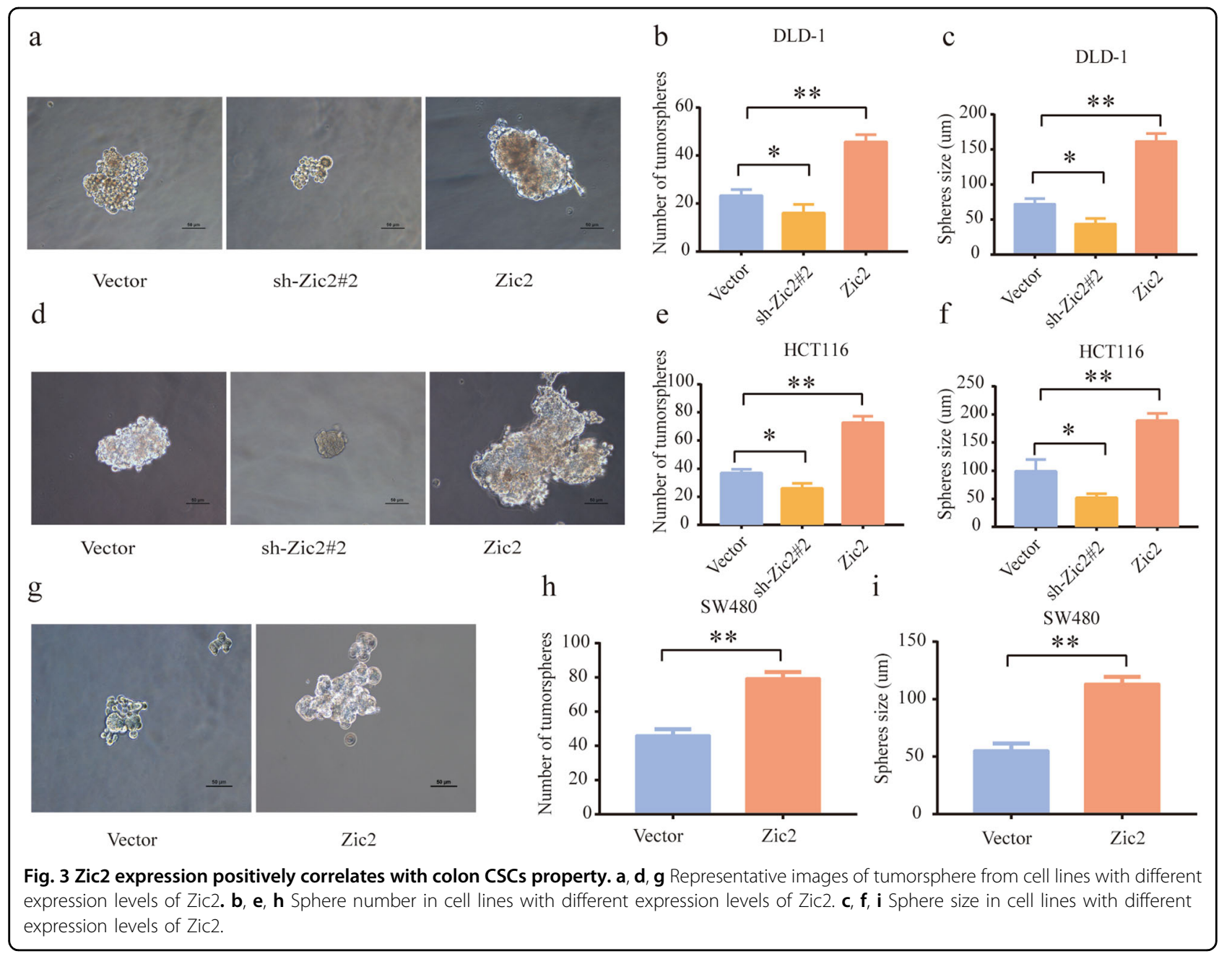

demonstrate that Zic2 promotes colon cancer proliferation and CSC properties. Wnt signaling is strongly linked to tumor proliferation and CSC properties ${ }^{2,3}$. Therefore, we hypothesized that Wnt signaling might be involved in Zic2-mediated colon cancer growth promotion. The TOP/FOP-Flash reporter assay further verified our hypothesis (Fig. 4a-c). Ectopic expression of Zic2 in DLD1 cells, as well as in HCT116 and SW480 cells, consistently increased luciferase activity, while Zic2 knockdown in DLD-1 and HCT116 cells decreased luciferase activity (all $P<0.05$ ).

Given that Zic2 mediates the cell cycle and CSC properties, cell cycle- and CSC-related proteins were assessed in our research. We found that Zic2 initiated the transcription of canonical downstream target genes of the Wnt pathway, such as cyclin D1, CD44, and Lgr $5^{15,16}$. In response to ectopic expression of Zic2, the expression of cyclin D1, CD44, and Lgr5 in DLD-1, HCT116, and SW480 cells was remarkably elevated at both the protein and mRNA levels (Fig. 4d-k). Correspondingly, Zic2 knockdown in DLD-1 and HCT116 cells markedly decreased the levels of cyclin D1, CD44, and Lgr5. Bioinformatic analysis via the GEPIA database (Supplementary Fig. 5a-c) and with our RNA sequencing results (Fig. 4l) further supported that Zic2 promotes the transcription of cyclin D1, CD44, and Lgr5.

In both cells with ectopic expression of Zic2 and cells with knockdown of Zic2, the transcript levels of $\beta$-catenin did not change. Moreover, the protein levels of total $\beta$-catenin and active $\beta$-catenin (Ser45) were elevated in response to ectopic expression of Zic2 in the DLD-1 and SW480 cell lines. Intriguingly, the levels of total $\beta$-catenin were not changed in the HCT116-Zic2 cell line. In addition, barely any active $\beta$-catenin protein could be detected in HCT116 cells due to the Ser45 mutation in this cell line ${ }^{20}$. We also found that the nuclear localization of $\beta$-catenin was unchanged in the HCT116-Zic2 cell line, but it was markedly increased in response to ectopic expression of Zic2 in the DLD-1 and SW480 cell lines (Fig. 4d, j). Taken together, the data show that Zic2 enhances Wnt signaling activity in colon cancer. 


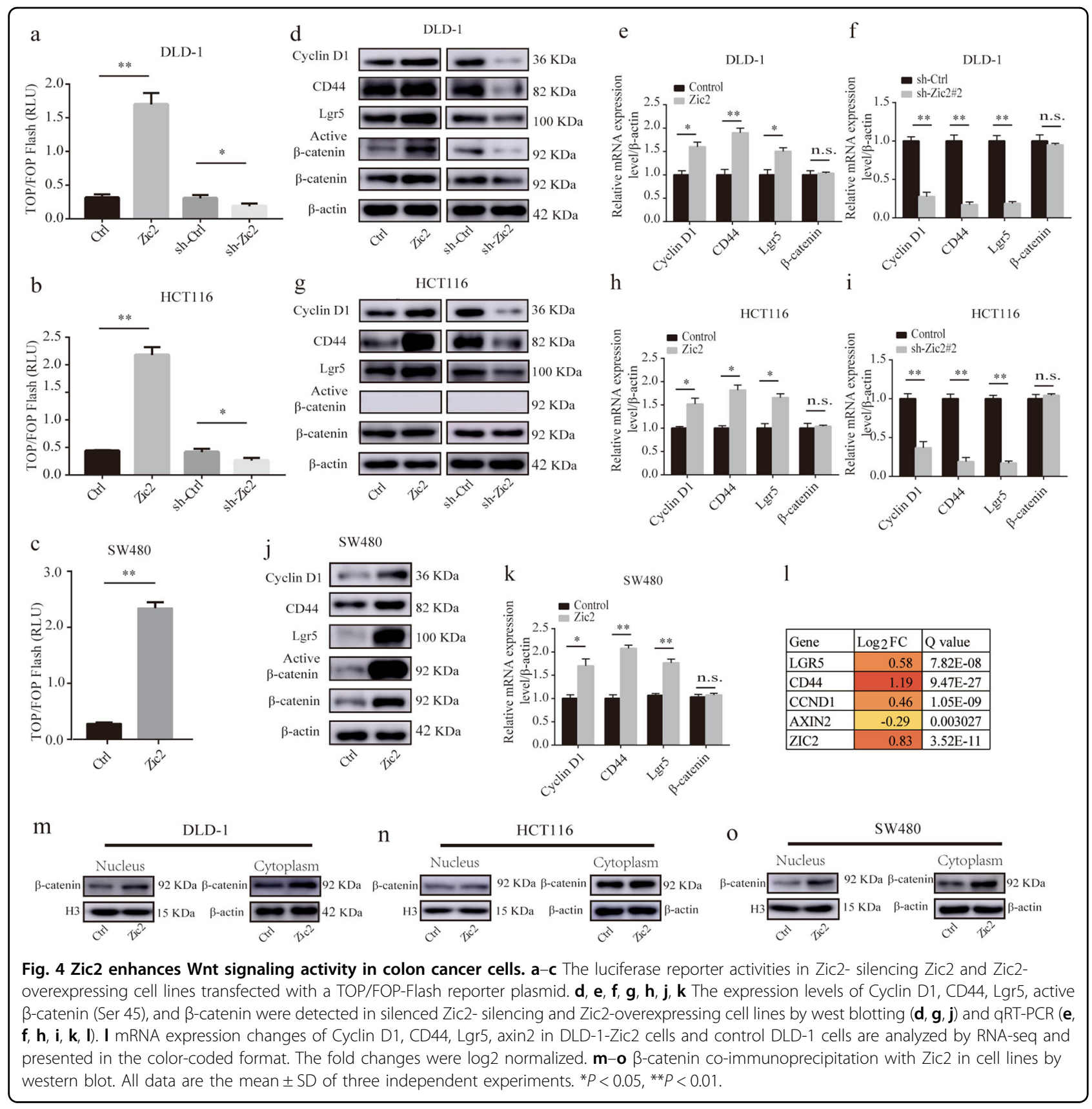

Zic2 activates Wnt signaling in colon cancer cells by interacting with $\beta$-catenin and repressing Axin2

GO molecular function analysis suggested that Zic2 might execute its biological function in colon cancer via DNA/protein binding and transcription regulator activity (Supplementary Fig. 4e). We first demonstrated that Zic2 interacted with $\beta$-catenin in DLD-1, SW480, and HCT116 cells to activate Wnt signaling (Fig. $5 \mathrm{a}-\mathrm{c}$ ). Because of the differences in $\beta$-catenin levels in colon cancer cells with modified Zic2 expression, especially the peculiar unchanged $\beta$-catenin levels in HCT116 cells, we hypothesized that disruption of the $\beta$-catenin destruction complex might be essential for the enhanced Wnt signaling activity induced by Zic2 in colon cancer. The results of qPCR (Supplementary Fig. 6a-e) and RNA sequencing (Fig. 4l) analysis showed that the transcript levels of Axin2 were remarkedly decreased in response to ectopic expression of Zic2. Western blotting assays also supported that enhancing Zic2 expression decreased Axin2 protein levels (Fig. 5d, e). Correspondingly, depletion of Zic2 reversed these changes (Fig. 5d-f). Collectively, these results indicate that Zic2 exerts multilevel 


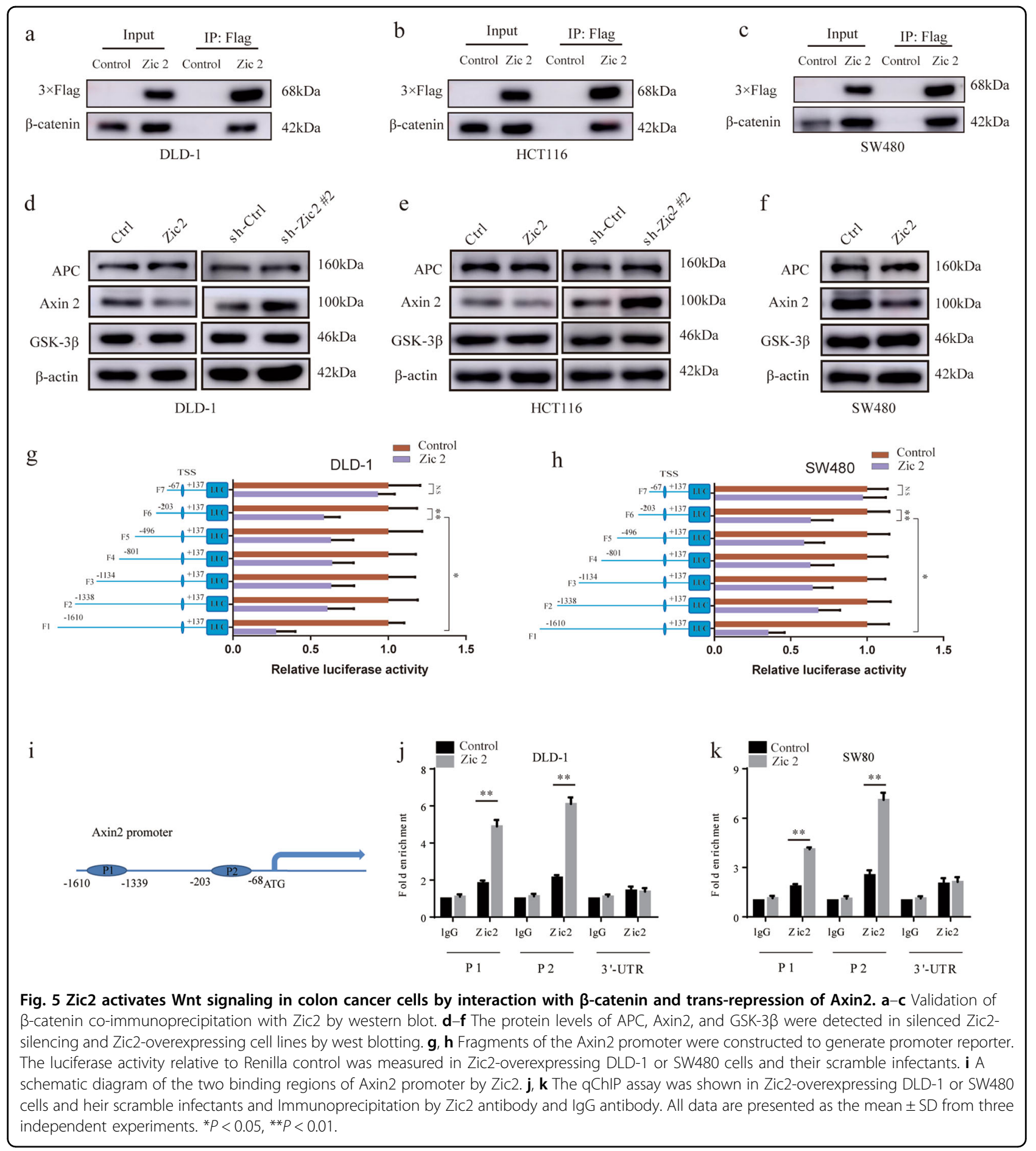

regulation on Wnt signaling through interaction with $\beta$-catenin and repression of Axin2 in colon cancer.

We further explored whether Zic2 activates the Wnt/ $\beta$-catenin pathway via transcriptional activation of Axin2. First, a full-length Axin2 promoter (from -1610 to $+137 \mathrm{bp})$ reporter construct and three other truncated constructs were constructed and transfected into DLDZic2 and SW480-Zic2 cells and control cells (Fig. 5g, h). The luciferase activity of the Axin2 promoter was detected by dual-luciferase reporter assay. Ectopic expression of Zic2 led to decreased luciferase activity of the fulllength fragments, while cells containing fragments with 


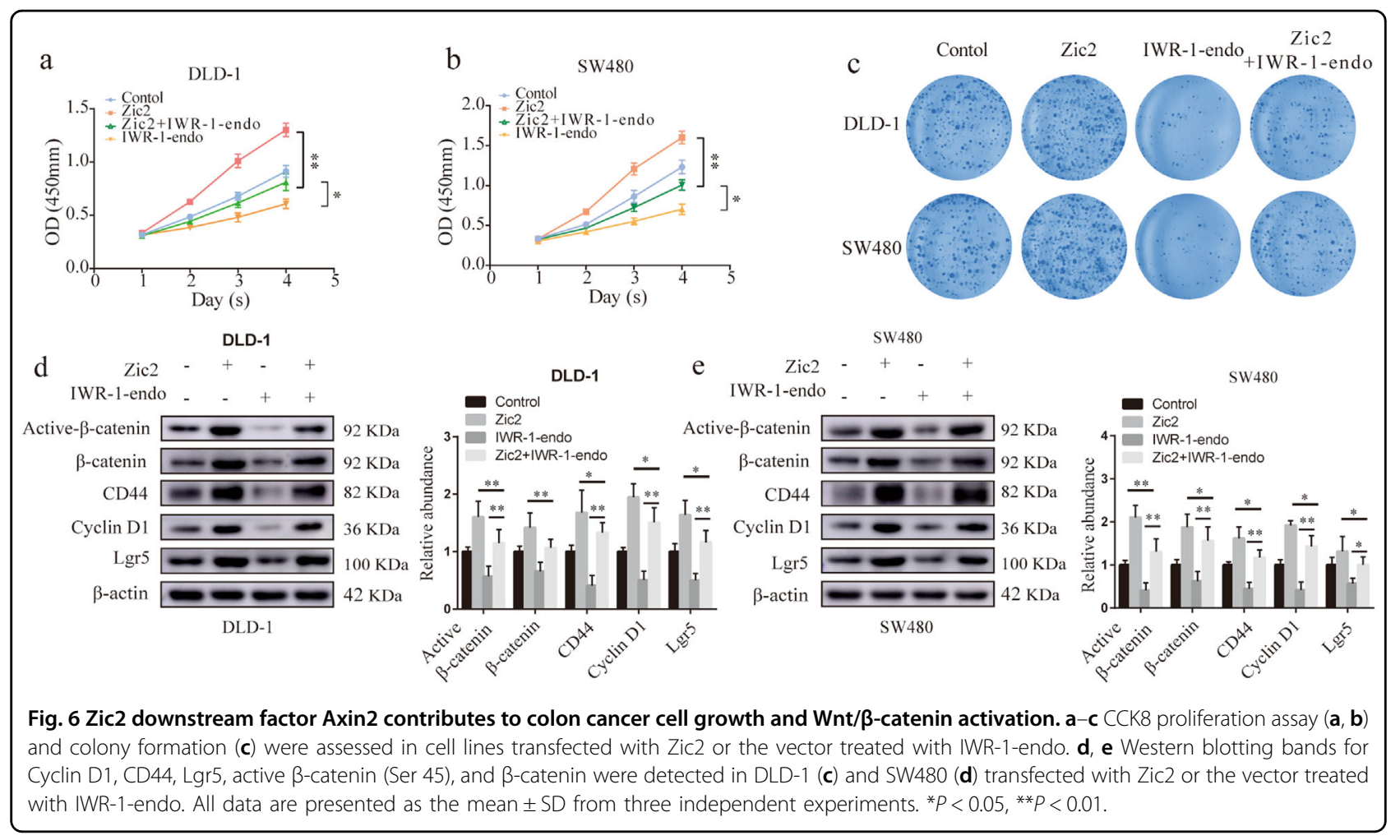

truncated fragments affecting residues from -1610 to $-1339 \mathrm{bp}$ (P1) and from -203 to -68 (P2) showed normal luciferase activity, suggesting that Zic2 could repress Axin2 expression by binding to the P1 and P2 fragments of the Axin2 promoter (Fig. 5i). Next, we attempted to confirm whether Zic2 binds to a unique site within the Axin 2 promoter in vivo by using a qChIP assay. Two pairs of primers were designed to amplify the four P1 and P2 fragments of the Axin2 promoter region. The results showed that ectopic expression of Zic2 enhanced the binding of Zic2 to the P1 and P2 fragments (Fig. 5j, k). All of these results indicate that Zic2 could bind to specific areas of the Axin2 promoter and transcriptionally activate Axin2 in colon cancer cells.

To further determine whether Axin2 is involved in Zic2-mediated Wnt signaling activation, we stabilized Axin2 using IWR-1-endo and further investigated its impact on the functions of Zic2 (Fig. 6a-c). Treating DLD-1-Zic2 and SW480-Zic2 cells with IWR-1-endo remarkably attenuated cell viability and colony formation in vitro. Furthermore, following the decrease in Axin2 levels induced by Zic2, Wnt/ $\beta$-catenin signaling was recovered, as visualized by elevated levels of $\beta$-catenin, cyclin D1, CD44, and Lgr5 (Fig. 6d, e). Taken together, these results led us to conclude that Zic2 promotes colon cancer growth by activating Wnt signaling via interaction with $\beta$-catenin and repression of Axin2.

\section{Correlation between Zic2 and Wnt signaling in vivo}

To validate the function of Zic2 in vivo, Zic2overexpressing colon cancer cells and negative control cells were subcutaneously injected into nude mice. Three weeks later, we measured the weight of xenograft tumors and found that ectopic expression of Zic2 significantly augmented the weight of xenograft tumors (Fig. 7a-f). By IHC analysis of the xenograft tumors, we found that Zic2 was positively associated with the expression of cyclin D1, CD44, and Lgr5, and negatively associated with the expression of Axin2 (Fig. 7g). The inverse correlation between Zic2 and Axin2 was further validated by IHC analysis of human colon cancer samples (Fig. 7h, i). Our data confirmed that Zic2 activated Wnt signaling by repressing Axin2 in vivo.

\section{Discussion}

Zic2 is upregulated and correlates with poor survival in various cancers, such as hepatocellular carcinoma ${ }^{30}$, clear cell renal cell carcinoma ${ }^{41}$, oral squamous cell carcinoma $^{42}$, and epithelial ovarian cancer ${ }^{43}$. In our study, a similar conclusion was drawn in colon cancer, and this is the first analysis of the relationship between Zic2 and prognosis using human colon cancer samples. However, Zic2 is barely expressed in breast cancer, in which high Zic2 expression predicts better survival. The reason for its positive relationship with prognosis in breast cancer is because in this cancer, Zic2 directly represses STAT3 


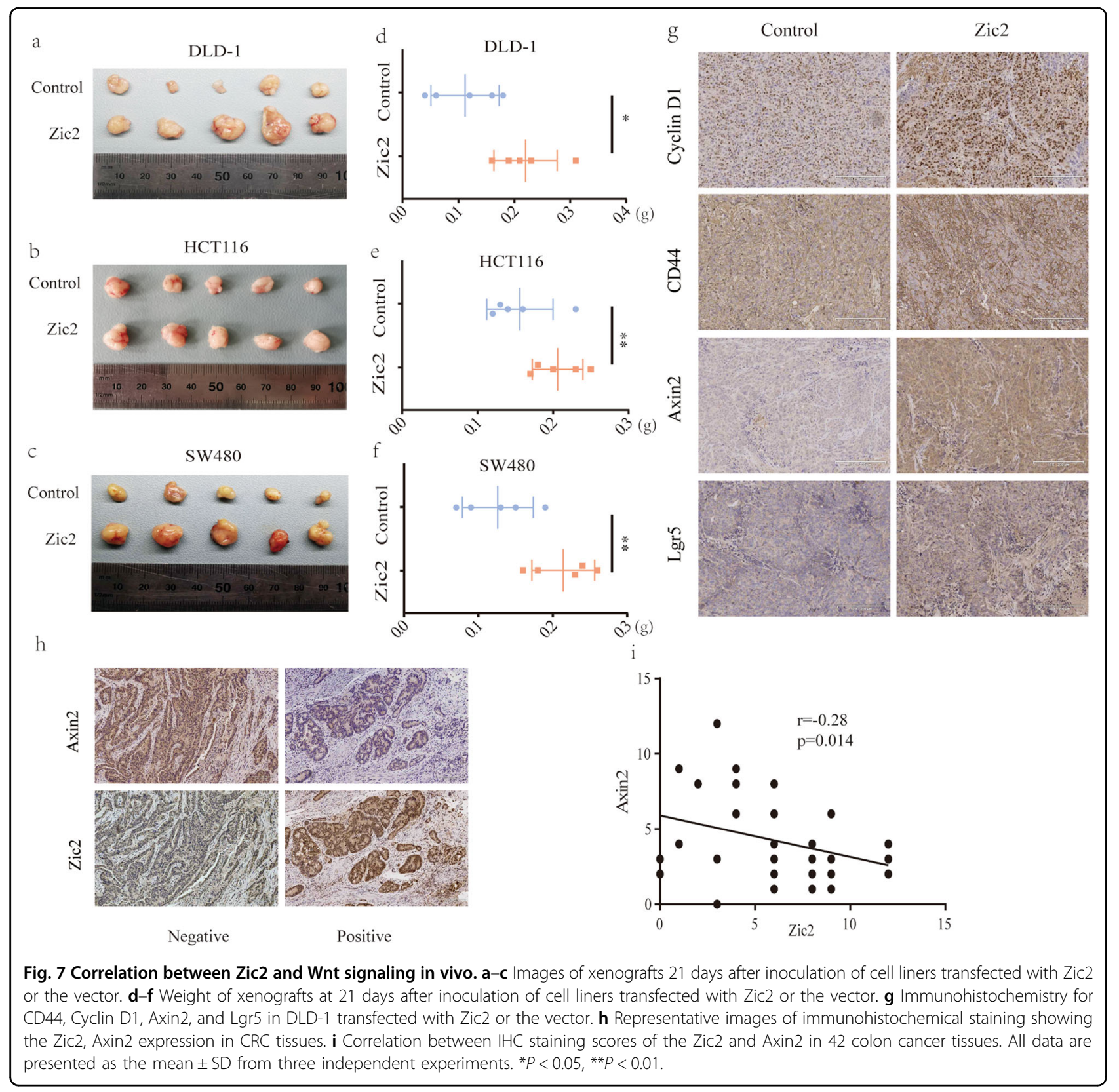

transcription $^{29}$. However, in most cancers, Zic2 acts as a transcriptional activator promoting tumorigenesis ${ }^{21,30,31}$. Intriguingly, we demonstrated for the first time that Zic2 acts as both a transcriptional coactivator and transcriptional repressor in colon cancer. Zic2 directly represses Axin2 transcription, which results in the disruption of the $\beta$-catenin destruction complex and the subsequent accumulation of active $\beta$-catenin in SW480 and DLD-1 cells. More intriguingly, ectopic expression of Zic2 in HCT116 cells did not change the level of active $\beta$-catenin, which might be due to the in-frame deletion at Ser45 in HCT116 cells ${ }^{20}$. Given that Zic2 activated Wnt/ $\beta$-catenin signaling in HCT116 cells, we speculated that there exists another mechanism of Zic2-mediated Wnt signaling activation. As expected, we confirmed that Zic2 interacts with $\beta$-catenin in SW480 and DLD-1 cell lines besides the HCT116 cell line $^{31}$. Zic2 might enhance Wnt/ $\beta$-catenin signaling in colon cancer via chromatin remodeling ${ }^{21,44,45}$. Collectively, in addition to directly repressing Axin2 transcription, Zic2 also interacts with $\beta$-catenin to activate Wnt signaling in colon cancer. Our study revealed multilevel regulation of Wnt signaling by Zic2 in colon cancer.

A previous study reported that Zic2 enhances Wnt signaling and plays crucial roles in tumorigenesis, and the same was found in our study ${ }^{31}$. However, limited downstream target genes affected by the Zic2-Wnt/ $\beta$-catenin 
axis have been identified, with only Glut1 being reported so $\mathrm{far}^{31}$. In the present study, we identified that Zic2 enhances Wnt signaling and initiates the transcription of several novel downstream target genes, including cyclin D1, CD44, and Lgr5, which promote proliferation and stemness in colon cancer cells.

A role of Zic2 in proliferation has not been conclusively demonstrated. Zic2 promotes proliferation in various cancers $^{30,31}$ but not in breast cancer ${ }^{29}$. Zic2 $2^{-1-}$ mice display a normal rate of proliferation ${ }^{46}$. Interestingly, Ishiguro et al. reported that phosphorylation of Zic2 at Ser200 decreases the interaction between Zic2 and RNA helicase in mice, which can suppress the transcriptional activation potential of Zic2 ${ }^{47}$. However, posttranslational modification and mutation of Zic2 cannot be excluded as reasons for our current findings, and the influence of these factors remains to be further evaluated. The discrepancy in its effects in different cancers makes it difficult to define the role of Zic2 in proliferation. In our study, ectopic expression of Zic2 promoted colon cancer proliferation by activating cyclin D1 transcription, in addition to cell cycle transition from G0/G1 to $S$ phase in vitro. Chandrasekaran et al. reported that silencing Zic2 expression in benign prostate epithelial cells inhibited anchorage-independent spheroid and tumor formation by repressing CD44, Sox2, and Notch1 expression, indicating that Zic2 might be closely associated with self-renewal properties ${ }^{26}$. A similar conclusion was made in liver cancer stem cells ${ }^{21}$. Herein, we identified for the first time that Zic2 accelerates sphere formation in vitro. Zic2 upregulated CD44 and Lgr5, well-known CSC mar$\operatorname{kers}^{14,48-52}$, via $W n t / \beta$-catenin signaling. Our study also revealed that Zic2 has effects on the maintenance of colon CSCs.

\section{Conclusions}

In summary, we demonstrated that Zic2 is upregulated in colon cancer samples and that high Zic2 expression correlates with unfavorable survival of colon cancer patients. Our results indicate that Zic2 promotes the proliferation and tumorigenesis of colon cancer. Zic2 functions in colon cancer, at least in part, by enhancing $\mathrm{Wnt} / \beta$-catenin signaling via collaboration with $\beta$-catenin and repressing Axin2. The above results suggest that the inhibition of Zic2 might be a potential therapeutic strategy for colon cancer patients.

\section{Acknowledgements}

We are grateful for the help of Dr. Shan Li. Thanks for the support of the Center for Translational Medicine of First Affiliated Hospital of Xi'an Jiaotong University.

\section{Author contributions}

Z.S.X., J.B.Z., and J.H.Y. performed the experiments, acquired data, and drafted the article. All authors revised the article critically for important intellectual content. J.G., C.C., and X.P.L. analyzed and interpreted data. Z.L.C., X.J.W., L.Y.Y., and C.Q. collected the clinical samples and evaluated all specimens. X.J.S. and J. H.Y. substantially contributed to conception and design. All authors read and approved the final manuscript and agreed to be accountable for all aspects of the research in ensuring that the accuracy or integrity of any part of the work are appropriately investigated and resolved.

\section{Funding}

This work was funded by a grant from the National Natural Science Foundation of China (Grant serial numbers: 81972720, 81101874, 81172362), the Coordinative and Innovative Plan Projects of the Science and Technology Program in Shaanxi Province (Grant serial number: 2013KTCQ03-08), the Science and Technology Project of Shaanxi Province (Grant serial numbers: 2016SF-015, 2019SF-065), the Fundamental Research Funds for the Central Universities (Grant serial number: xjj2018123).

\section{Data availability}

The datasets generated and/or analyzed during the current study are not publicly available but are available from the corresponding author on reasonable request.

\section{Consent for publication}

All authors consent for publication.

\section{Ethics approval and consent to participate}

All animal experiments in our study were carried out in accordance with the Helsinki Declaration, and approved by the Ethics Committee of The First Affiliated Hospital of Xi'an Jiaotong University. Patients were informed that the resected specimens were stored by the hospital and potentially used for scientific research, and that their privacy would be maintained. All patients provided informed consent prior to undergoing surgery. Our study protocol was approved by the Ethics Committee of the First Affiliated Hospital of Xi'an Jiaotong University.

\section{Conflict of interest}

The authors declare no competing interests.

\section{Publisher's note}

Springer Nature remains neutral with regard to jurisdictional claims in published maps and institutional affiliations.

Supplementary information The online version contains supplementary material available at https://doi.org/10.1038/s41419-021-03863-w.

Received: 3 March 2021 Revised: 18 May 2021 Accepted: 19 May 2021 Published online: 07 June 2021

\section{References}

1. Siegel, R. L., Miller, K. D. \& Jemal, A. Cancer statistics, 2019. CA Cancer J. Clin. 69, 7-34 (2019).

2. Basu, S., Haase, G. \& Ben-Ze'ev, A. Wnt signaling in cancer stem cells and colon cancer metastasis. F1000Res 5 (2016).

3. Nusse, R. \& Clevers, H. Wnt/ $\beta$-catenin signaling, disease, and emerging therapeutic modalities. Cell 169, 985-999 (2017).

4. Zhan, T., Rindtorff, N. \& Boutros, M. Wht signaling in cancer. Oncogene $\mathbf{3 6}$ 1461-1473 (2017).

5. Cancer Genome, Atlas, N. Comprehensive molecular characterization of human colon and rectal cancer. Nature 487, 330-337 (2012).

6. Kimelman, D. \& Xu, W. beta-catenin destruction complex: insights and questions from a structural perspective. Oncogene 25, 7482-7491 (2006).

7. Amit, S. et al. Axin-mediated CKI phosphorylation of beta-catenin at Ser 45: a molecular switch for the Wnt pathway. Genes Dev. 16, 1066-1076 (2002).

8. Conacci-Sorrell, M., Zhurinsky, J. \& Ben-Ze'ev, A. The cadherin-catenin adhesion system in signaling and cancer. J. Clin. Invest. 109, 987-991 (2002).

9. Yamamoto, $\mathrm{H}$. et al. Phosphorylation of axin, a Wnt signal negative regulator, by glycogen synthase kinase-3beta regulates its stability. J. Biol. Chem. 274, 10681-10684 (1999). 
10. Behrens, J. et al. Functional interaction of an axin homolog, conductin, with beta-catenin, APC, and GSK3beta. Science 280, 596-599 (1998).

11. Kishida, S. et al. Axin, a negative regulator of the wnt signaling pathway, directly interacts with adenomatous polyposis coli and regulates the stabilization of beta-catenin. J. Biol. Chem. 273, 10823-10826 (1998).

12. Lee, E., Salic, A., Kruger, R., Heinrich, R. \& Kirschner, M. W. The roles of APC and Axin derived from experimental and theoretical analysis of the Wnt pathway. PLOS Biol. 1, E10 (2003).

13. Li, S., Wang, C., Liu, X., Hua, S. \& Liu, X. The roles of AXIN2 in tumorigenesis and epigenetic regulation. Fam. Cancer 14, 325-331 (2015).

14. He, Y. et al. CDCP1 enhances Wnt signaling in colorectal cancer promoting nuclear localization of $\beta$-catenin and E-cadherin. Oncogene 39, 219-233 (2020).

15. Taciak, B., Pruszynska, I., Kiraga, L., Bialasek, M. \& Krol, M. Wnt signaling pathway in development and cancer. J. Physiol. Pharmacol. 69, 185-196 (2018).

16. Yamamoto, Y. et al. Overexpression of orphan G-protein-coupled receptor, Gpr49, in human hepatocellular carcinomas with beta-catenin mutations. Hepatology 37, 528-533 (2003).

17. Mazzoni, S. M. \& Fearon, E. R. AXIN1 and AXIN2 variants in gastrointestinal cancers. Cancer Lett. 355, 1-8 (2014).

18. Leung, J. Y. et al. Activation of AXIN2 expression by beta-catenin-T cell factor. A feedback repressor pathway regulating Wnt signaling. J. Biol. Chem. 277, 21657-21665 (2002).

19. Novellasdemunt, L., Antas, P. \& Li, V. S. W. Targeting Wnt signaling in colorectal cancer. A review in the theme: cell signaling: proteins, pathways and mechanisms. Am. J. Physiol., Cell Physiol. 309, C511-C521 (2015).

20. Ilyas, M., Tomlinson, I. P., Rowan, A., Pignatelli, M. \& Bodmer, W. F. Beta-catenin mutations in cell lines established from human colorectal cancers. Proc. Natl Acad. Sci. USA 94, 10330-10334 (1997).

21. Zhu, P. et al. ZIC2-dependent OCT4 activation drives self-renewal of human liver cancer stem cells. J. Clin. Invest. 125, 3795-3808 (2015).

22. Pourebrahim, R. et al. Transcription factor Zic2 inhibits Wnt/beta-catenin protein signaling. J. Biol. Chem. 286, 37732-37740 (2011).

23. Nagai, T. et al. Zic2 regulates the kinetics of neurulation. Proc. Natl Acad. Sci. USA 97, 1618-1623 (2000).

24. Brewster, R., Lee, J. \& Ruiz i Altaba, A. Gli/Zic factors pattern the neural plate by defining domains of cell differentiation. Nature 393, 579-583 (1998).

25. Nakata, K., Nagai, T., Aruga, J. \& Mikoshiba, K. Xenopus Zic family and its role in neural and neural crest development. Mech. Dev. 75, 43-51 (1998).

26. Chandrasekaran, B. et al. Chronic exposure to cadmium induces a malignant transformation of benign prostate epithelial cells. Oncogenesis 9, 23 (2020).

27. Chan, D. W. et al. Zic2 synergistically enhances Hedgehog signalling through nuclear retention of Gli1 in cervical cancer cells. J. Pathol. 225, 525-534 (2011).

28. Zhang, P., Yang, F., Luo, Q., Yan, D. \& Sun, S. miR-1284 inhibits the growth and invasion of breast cancer cells by targeting ZIC2. Oncol. Res 27, 253-260 (2019).

29. Liu, Z.-H. et al. ZIC2 is downregulated and represses tumor growth via the regulation of STAT3 in breast cancer. Int. J. Cancer 147, 505-518 (2020).

30. Lu, S. X. et al. Zic2 promotes tumor growth and metastasis via PAK4 in hepatocellular carcinoma. Cancer Lett. 402, 71-80 (2017).

31. Zhao, Z. et al. beta-Catenin/Tcf7l2-dependent transcriptional regulation of GLUT1 gene expression by Zic family proteins in colon cancer. Sci. Adv. $\mathbf{5}$, eaax0698 (2019).
32. Huang, S. \& Jin, A. ZIC2 promotes viability and invasion of human osteosarcoma cells by suppressing SHIP2 expression and activating PI3KAKT pathways. J. Cell Biochem. 119, 2248-2257 (2018).

33. $\mathrm{Yu}$, J. et al. CDX2 inhibits the proliferation and tumor formation of colon cancer cells by suppressing Wnt/ $\beta$-catenin signaling via transactivation of GSK$3 \beta$ and Axin2 expression. Cell Death Dis. 10, 26 (2019).

34. Li, R., Li, Y., Kristiansen, K. \& Wang, J. SOAP: short oligonucleotide alignment program. Bioinformatics 24, 713-714 (2008).

35. Kim, D., Langmead, B. \& Salzberg, S. L. HISAT: a fast spliced aligner with low memory requirements. Nat. Methods 12, 357-360 (2015).

36. Langmead, B. \& Salzberg, S. L. Fast gapped-read alignment with Bowtie 2. Nat Methods 9, 357-359 (2012).

37. Li, B. \& Dewey, C. N. RSEM: accurate transcript quantification from RNA-Seq data with or without a reference genome. BMC Bioinforma. 12, 323 (2011).

38. Kolde, R. Package 'pheatmap'. 2019-01-04 13:50:12 UTC.

39. Love, M. I., Huber, W. \& Anders, S. Moderated estimation of fold change and dispersion for RNA-seq data with DESeq2. Genome Biol. 15, 550 (2014).

40. Abdi, H. Bonferroni and Šidák corrections for multiple comparisons. Encycl. Meas. Stat. 1, 1-9 (2007).

41. Shang, Z. et al. The level of zinc finger of the cerebellum 2 is predictive of overall survival in clear cell renal cell carcinoma. Transl. Androl. Urol. 9, 614-620 (2020).

42. Sakuma, $\mathrm{K}$. et al. Expression status of Zic family member 2 as a prognostic marker for oral squamous cell carcinoma. J. Cancer Res. Clin. Oncol. 136, 553-559 (2010)

43. Marchini, S. et al. The zinc finger gene ZIC2 has features of an oncogene and its overexpression correlates strongly with the clinical course of epithelial ovarian cancer. Clin. Cancer Res. 18, 4313-4324 (2012).

44. Ishiguro, A., Ideta, M., Mikoshiba, K., Chen, D. J. \& Aruga, J. ZIC2-dependent transcriptional regulation is mediated by DNA-dependent protein kinase, poly (ADP-ribose) polymerase, and RNA helicase A. J. Biol. Chem. 282, 9983-9995 (2007).

45. Frank, C. L. et al. Regulation of chromatin accessibility and Zic binding at enhancers in the developing cerebellum. Nat. Neurosci. 18, 647-656 (2015).

46. Elms, P., Siggers, P., Napper, D., Greenfield, A. \& Arkell, R. Zic2 is required for neural crest formation and hindbrain patterning during mouse development. Dev. Biol. 264, 391-406 (2003).

47. Ishiguro, A. \& Aruga, J. Functional role of Zic2 phosphorylation in transcriptional regulation. FEBS Lett. 582, 154-158 (2008).

48. Shimokawa, M. et al. Visualization and targeting of LGR5(+) human colon cancer stem cells. Nature 545, 187-192 (2017).

49. Zeki, S. S., Graham, T. A. \& Wright, N. A. Stem cells and their implications for colorectal cancer. Nat. Rev. Gastroenterol. Hepatol. 8, 90-100 (2011).

50. de Sousa e Melo, F. et al. A distinct role for Lgr5 stem cells in primary and metastatic colon cancer. Nature 543, 676-680 (2017).

51. Saigusa, S. et al. Clinical significance of LGR5 and CD44 expression in locally advanced rectal cancer after preoperative chemoradiotherapy. Int. J. Oncol. 41 1643-1652 (2012)

52. Gregorieff, A., Liu, Y., Inanlou, M. R., Khomchuk, Y. \& Wrana, J. L. Yap-dependent reprogramming of $\operatorname{Lgr} 5(+)$ stem cells drives intestinal regeneration and cancer. Nature 526, 715-718 (2015). 